\title{
Multi-level Brokering Solution for Interoperating Service and Desktop Grids`
}

\author{
Attila Kertész, Zoltán Farkas, and Péter Kacsuk \\ MTA SZTAKI Computer and Automation Research Institute, \\ H-1518 Budapest, P.O. Box 63, Hungary \\ \{attila.kertesz, zfarkas, kacsuk\}@sztaki.hu
}

\begin{abstract}
User communities have gathered around various Grid systems forming separate islands that represent borders they cannot cross. As these communities are growing and demanding more and more computational power, uniting these islands draws more attention in Grid research and development. This problem is called the Grid Interoperability problem. This issue may be tackled at different levels in the Grid middleware, this paper targets the level of Grid resource management also called as Grid brokering. This paper introduces a meta-brokering approach that means a higher level resource management by enabling automatic and simultaneous utilization of various Grid brokers managing resources of different Grid systems. Desktop Grids are using not only different interfaces, but also different technologies. Here we also introduce a novel solution to access Desktop Grids through specific brokers that can be managed by our Meta-Broker. In this way with our multilevel brokering solution we can unify several Service and Desktop Grids without modifying their implementation, policies or interfaces, providing the greatest computational power possible for all Grid user communities.
\end{abstract}

Keywords: Grid Interoperability, Grid Interoperation, Grid Resource Management, Grid Meta-brokering.

\section{Introduction}

E-Science infrastructures play an important role in enabling large-scale scientific research. In order to establish such e-infrastructures, various Grid systems have been created and run as a service for the scientific community. Originally, the aim of Grid systems was that anyone (called donors) could offer resources for the Grid, and anyone (called users) could claim resources dynamically, according to their actual needs, in order to solve a computational or data intensive task. This twofold aim has however not fully been achieved, and today we can observe two different trends in the development of Grid systems: Service Grids and Desktop Grids. In Service Grids, computational resources are offered as Grid services

\footnotetext{
* The research leading to these results has received funding from the European Community's Seventh Framework Programme FP7/2007-2013 under grant agreement 215483 (S-Cube) and from EDGI (RI-261556).
} 
that can be accessed by a large number of users. A resource can become part of the Grid by installing a predefined software set, or middleware. The middleware is, however, so complex that it often requires extensive effort to maintain. On the other hand, Desktop Grids are commonly known as volunteer computing systems, because they often rely upon the general public to donate compute resources, or spare cycles. Unlike Service Grids, which are based on complex architectures, volunteer computing has a simple architecture and has demonstrated the ability to integrate dispersed, heterogeneous computing resources with ease, successfully scavenging cycles from tens of thousands of idle desktop computers. The Grid research community considers Desktop Grids only as particular and limited forms of e-infrastructures, because they cannot work as services nor be used by anyone who has not already setup their projects to function in this environment. Additionally, unlike most Service Grids, which have reciprocal agreements for resource utilization among partners, participants in Desktop Grid systems, cannot use the system for their own goals. Until now, these two kinds of Grid systems have been completely separated, hence there has not been a mechanism to exploit their advantageous, individual features in a unified environment. However, with the objective to support new scientific communities who need extremely large numbers of resources, the solution could be to interconnect these two kinds of Grid systems into an integrated Service Grid - Desktop Grid infrastructure. User communities have gathered around various Grid systems (including Service and Desktop Grids) forming separate islands that represent borders they cannot cross. As these communities are growing and demanding more and more computational power, uniting these islands draws more attention in Grid research and development, which is called the Grid Interoperability problem [8]. Grid Resource Management tools evolved from manual discovery and task submission to sophisticated brokering solutions. To ease the simultaneous utilization of different Service Grids, researchers have started to revise current brokering solutions by extending existing resource brokers with multiple middleware support. In this paper we introduce a meta-brokering approach, which acts as a higher level brokering service by enabling automatic and simultaneous utilization of various Grid brokers managing resources of different Grid systems. Since Desktop Grids are using different technologies and lacking such brokers Service Grids have, here we introduce a novel solution to access Desktop Grids through specific gateways that act as service brokers and can be managed by our meta-brokering solution. In this way we can unify several Service and Desktop Grids without modifying their implementation, policies or interfaces, providing the greatest computational power possible for all user communities.

The rest of the paper is organized as follows. In Section 2 we present the related work for interoperation research with high-level brokering directions. In Section 3, we present a meta-brokering solution for service Grids, and in Section 4. we show how the Desktop Grids can be interfaced to meta-brokering with a novel service called 3G Bridge. In Section 5 we describe the multi-level brokering solution for Grid interoperation. 


\section{Related Work}

The problem of Grid interoperability and interoperation is a crucial issue to solve [8]. Meta-brokering approaches seek for interoperable solutions at the level of Grid resource management by enabling a higher level brokering solution that schedules user jobs among various Grid brokers/domains. Some of these metabrokering solutions, such as the meta-scheduling project in LA Grid [9], the delegated matchmaking with Koala 4 and decentralized scheduling with Gridway [3, aim at enabling communication among existing resource brokers in a sense that different domains are being examined as a whole, but they rather delegate resource information among domains, broker instances or gateways through their own, implementation-dependent interfaces. Usually the local domain has preference, and when a job is forwarded, the result should be transferred back to the initial instance. On the other hand, the advantage of our proposed metabrokering concept is that it does not require any modification of the existing Grid resource managers, since it utilizes and delegates broker information by reaching them through their current interfaces.

Regarding Grid interoperability solutions achieved so far, they are based on short-term solutions. The paper in [8] focuses on different Grid components (like job management, data management, information systems), and urges the usage of standards-based solutions. The interoperability solution for UNICORE and Globus described in [7] uses a translation mechanism to allow the execution of jobs submitted to UNICORE on Globus. In paper [2], authors show two solutions for using Condor resources in an OGSA-based infrastructure. The first option hides the details of the Condor system: job submission, job execution management and resource information providers are offered for the OGSA Grid to hide Condor details. On the other hand, the second option embeds OGSA within the Condor framework in order to provide controlled access to the Condor resources. These solutions target low-level interoperability of service Grids. Interoperability of service and Desktop Grids has been solved within the EDGeS [1] project with the help of the Generic Grid-Grid (3G) Bridge technology [10]. The architecture of $3 \mathrm{G}$ Bridge has been proven to be generic enough to easily solve interoperability of a number of service (gLite, ARC, Unicore) and Desktop (BOINC, XtremWeb, OurGrid) Grid systems. In this paper we introduce an extension of the $3 \mathrm{G}$ Bridge that allows us using it as part of our meta-brokering approach, that helps to solve Grid interoperability at higher levels.

\section{Meta-Brokering for Service Grids}

In order to access resources of different Grids simultaneously, we use a metabrokering approach. The Grid Meta-Broker Service (GMBS) 6] is a higher level tool that matches resource brokers to user requests. The system is implemented as a web-service (WS), which is independent from middleware-specific components. In the following we describe the role of the components (depicted in Figure 1) and their interaction. The Translator components of GMBS are responsible 
for translating the resource specification defined by the user to the language of the appropriate resource broker that the meta-broker selects to invoke for a given job. We also use an extendable Broker Property Description Language (BPDL) [6] to express metadata about brokers. The Information Collector (IC) component of the GMBS stores the data of the reachable brokers and historical data of the previous submissions. During broker utilization the successful submissions and failures are tracked, and regarding these events a rank is modified for each special attribute in the BPDL of the appropriate broker. In this way, the BPDL documents represent and store the dynamic states of the brokers. When a large number of jobs with similar requirements are sent to GMBS, the so-called best effort matchmaking (choosing the less loaded one) may flood a broker and its utilized resources. To cope with this problem, there is an IS Agent (IS stands for Information System) service reporting to IC, which regularly checks and stores the load of the Grids of each connected broker.

The Invoker components are used to contact the brokers. Therefore the Invokers are broker-specific components: they communicate with the interconnected brokers, calling them with job requests and collecting the results. The user has to upload the job, Grid certificate proxies and input files along with the job description (JSDL) to the GMBS, and the Matchmaker component tries to find a proper broker for the request. If it could not find a broker that would be able to fulfil the user requirements, the request is discarded, otherwise the JSDL is translated to the language of the selected broker. In the JSDL extension the middleware constraint fields can be used to specify certificate proxy names for Grids/VOs. This information is used by the Invokers to select the valid certificate proxy from the uploaded files for the actual job submission. Then the responsible Invoker takes care of transferring the necessary files to the selected Grid environment. After job submission, it stages back the output files and upgrades the historical data stored in the Information Collector with the log of the utilized broker. The Core component of the service is responsible for managing the communication (information and data exchange) among the other components. Generally the following operations can be done through this interface: adding a new broker with BPDL, querying the available brokers and the name of the tracked Grids/VOs, adding new Information Systems to be tracked, submitting jobs and signaling submitted job results.

\section{3G Bridge: A Service Broker for Desktop Grids}

The Generic Grid-Grid Bridge (3G Bridge) has been created within the scope of the EDGeS project to solve Grid interoperability. The aim of 3G Bridge is to offer a generic implementation for a gateway service that allows the execution of jobs within different Grid middleware using Grid plugins. On the other hand, it offers a very simple web service interface for submitting jobs into its internal job database. An overview of the 3G Bridge architecture can be seen in Figure [1. The central component of the $3 \mathrm{G}$ Bridge is the $3 G$ Bridge Job Database. This database is responsible for storing data of jobs and plugins. The design is 


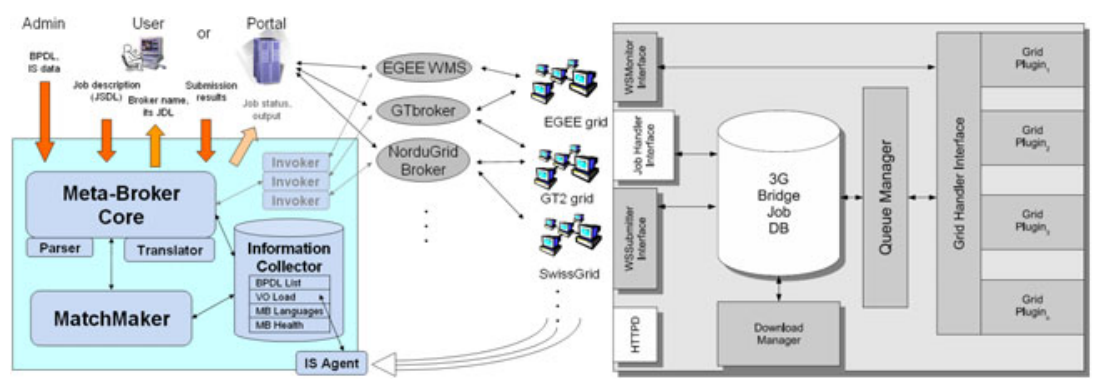

Fig. 1. The Grid Meta-Broker Service for Service Grids (on the left) and 3G Bridge for Desktop Grids (on the right)

very simple, for every job the following minimal set of attributes is kept track of: the internal identifier of the job, the name of the destination plugin, the name of the executable, the status of the job, the Grid identifier of the job, the command-line arguments of the job, the timestamp when the job has been added to the database, the list of input files and their locations and finally the list of output files (and their locations given that they have been produced). Based on this information, the Queue Manager interacts with the Grid Plugins through the generic Grid Handler Interface. The Grid Plugins have to implement some functions of the interface in order to create a working plugin: submit a set of jobs, update the status of all jobs belonging to the plugin, and poll the status of one job. These functions are responsible for managing jobs assigned to the given Grid plugin. Using this design we managed to create Grid plugins for gLite, BOINC, XtremWeb, OurGrid and BES-compatible resources. Clients of the 3G Bridge have two possibilities to submit new jobs to a new plugin: the WSSubmitter Interface and the Job Handler Interface. The WSSubmitter interface is a web service-based interface, offering very simple operations: submit - to send a set of jobs to $3 \mathrm{G}$ Bridge, the response of the submission operation is the list of $3 \mathrm{G}$ Bridge job identifiers assigned to the jobs; getStatus - to query the status of jobs from the $3 \mathrm{G}$ Bridge, the response is the sequence of job statuses; delete to cancel jobs; getOutput - to get the list of names and URLs of output files produced by jobs; getFinished - to get the list of job identifiers assigned to a Grid plugin that have finished; and finally getVersion - to get the version of the $3 \mathrm{G}$ Bridge service.

The Download Manager and HTTPD components have responsible for managing input and output files of jobs. The Download Manager is responsible for fetching input files of submitted jobs onto the $3 \mathrm{G}$ Bridge server machine. The HTTP component is a simple web server that makes 3G Bridge clients able to fetch output files produced by $3 \mathrm{G}$ Bridge jobs. All this information makes $3 \mathrm{G}$ Bridge usable as a Grid gateway, but the information needed for brokering is not propagated yet. In order to make 3G Bridge usable for our meta-brokering solution, the WSMonitor Interface has been created. This is a web service interface offering basic information about Grid plugins: getRunningJobs returns the number of jobs in the running status within the Grid belonging to a Grid 
plugin, getWaitingJobs returns the number of jobs in the waiting status within the Grid belonging to a Grid plugin, and getCPUCount returns the total number of usable CPUs within the Grid belonging to the Grid plugin. It is important to emphasise that the provided information does not reflect the status of the 3G Bridge Job Database, but the status of the Grid connected to the given plugin. This difference is clearly indicated in Figure 11. With the above in mind, we can state that a $3 \mathrm{G}$ Bridge service can offer every necessary interface and information so that the GMBS can schedule jobs onto it. In case of BOINC, the above information is calculated based on the BOINC project's database the following way: the number of running jobs comes from the result table of the BOINC database. The number of waiting jobs is determined in the same way, the difference is the value the query looks for. The CPU count is collected from the host table of the BOINC database. The query summarizes the number of CPUs of the hosts, whose last connection was within the last 24 hours, thus the host is assumed to be active.

\section{Meta-brokering among Service and Desktop Grids}

In order to bring Desktop Grid resources to our meta-brokering environment presented in Section 3, we use the 3G Bridge described in Section 4 . The GMBS sees $3 \mathrm{G}$ Bridges as service brokers, therefore we have created a new Invoker that uses the WSSubmitter web-service interface of the 3G Bridge (described by a WSDL). The IS Agent of GMBS is also interfaced to the WSMonitor webservice interface of the $3 \mathrm{G}$ Bridge. Through this interface the GMBS can be informed about the number of running and waiting jobs, and also about the number of available CPUs in the actual Desktop Grid. Exactly these attributes are used by the IS Agent to store the actual load information in the BPDL descriptions of the utilized brokers by GMBS. The Translator component has also been extended to convert the JDSL description of the job provided by the user to the XML description needed by the $3 \mathrm{G}$ Bridge (which contains the name of the executable, arguments, possible input/output files and the name of the target Desktop Grid).

Regarding security issues, in Section 3 we have stated that the Invokers use the attached proxies named in the JSDL extension of the submitted job for authentication by the selected service broker and other related Grid services. Additionally, the GMBS makes use of the EDGeS Application Repository [1]: this service contains application descriptions. For each application, the hash of the executable and supporting Desktop Grid resources are stored within the repository. Eg. in BOINC, for a given application we can collect the set of BOINC projects that support the application based on its hash. With the EDGeS Application Repository the Meta-Broker service can have an overview of which application is supported by which BOINC project, and selects a $3 \mathrm{G}$ Bridge service during scheduling if and only if the given application is supported by the given BOINC project. To enable this validation, the IS Agent has been extended to periodically fetch the contents of the Application Repository as there could be 
updates, and stores the MD5 hashes of the available applications in the BPDL of the appropriate $3 \mathrm{G}$ Bridge. The validation is done during matchmaking: the MD5 hash of the application to be scheduled is compared to the ones found in the repository and stored in the BPDLs. If the validation fails for a given 3G Bridge (which means that the job is not supported by the Desktop Grid reachable by the $3 \mathrm{G}$ Bridge), the $3 \mathrm{G}$ Bridge is filtered out from the candidate brokers.

In general, the matchmaking process of GMBS remains the same: the list of available brokers are filtered according to the special requirements of the submitted job, then the actual load and performance data stored in the BPDLs of the brokers (including $3 \mathrm{G}$ Bridges) are used to select the best candidate. In this way at the meta-level the GMBS schedules user jobs among brokers of different Grids, then the invoked broker schedules the job among resources in its Grid environment. The extended meta-brokering solution is depicted in Figure 2. which serves as an interoperable brokering service among both Service and Desktop Grids.

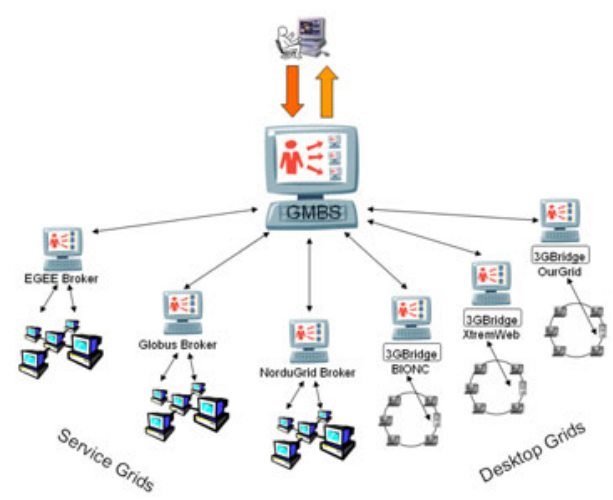

Fig. 2. Interoperation between Service and Desktop Grids with meta-brokering

\section{Conclusions}

Grid interoperability and interoperation among different Grid systems gets more and more attention due to the increasing number of users and Grid applications. Though some low level solutions for interconnecting different middleware systems have already appeared, a higher level brokering solution was still missing. In this paper we have shown how the Grid Meta-Broker Service can serve as an interoperable service to interconnect several Service and Desktop Grids. The design and the architecture of the GMBS enable a multi-level interoperable brokering by utilizing existing resource brokers of different Grid systems. It gathers and utilizes meta-data about existing widely used brokers from various Service Grid systems, and communicates with $3 \mathrm{G}$ Bridge instances to reach resources of Desktop Grid systems in a brokered way. The novel advantage of our extended 
meta-brokering concept is that it does not require any modification of the existing Grid systems, since it gathers and delegates broker-related information and utilizes them through their current interfaces. In this way the presented multilevel brokering solution can unify several Service and Desktop Grids without modifying their implementation, policies or interfaces, providing the greatest computational power possible for all Grid user communities. Several ideas of the described meta-brokering and the $3 \mathrm{G}$ Bridge solution have built into the $\mathrm{P}$ GRADE and WS-PGRADE portal that provide workflow level interoperability among various gLite, GT2, GT4 and BOINC Grids 5. Our future work aims at investigating different scheduling policies for the coordinated use of these heterogeneous resources, and extending our meta-brokering solution to Cloud infrastructures.

\section{References}

1. Balaton, Z., Farkas, Z., Gombás, G., Kacsuk, P., Lovas, R., Marosi, A., Emmen, A., Terstyánszky, G., Kiss, T., Kelley, I., Taylor, I., Lodygensky, O., Cardenas-Montes, M., Fedak, G., Araujo, F.: EDGeS: the common boundary between service and desktop grids. Parallel Processing Letters 18(3), 433-445 (2008)

2. Chapman, C., Wilson, P., Tannenbaum, T., Farrellee, M., Livny, M., Brodholt, J., Emmerich, W.: Condor services for the Global Grid: interoperability between Condor and OGSA. In: Proceedings of the 2004 UK E-Science All Hands Meeting, Nottingham, UK, pp. 870-877 (2004)

3. Leal, K., Huedo, E., Llorente, I.M.: A decentralized model for scheduling independent tasks in Federated Grids. Future Generation Computer Systems 25(8), 840-852 (2009)

4. Iosup, A., Epema, D.H.J., Tannenbaum, T., Farrellee, M., Livny, M.: InterOperating Grids through Delegated MatchMaking. In: Proceedings of the International Conference for High Performance Computing, Networking, Storage and Analysis (SC 2007), Reno, Nevada (November 2007)

5. Kacsuk, P., Karóczkai, K., Hermann, G., Sipos, G., Kovács, J.: WS-PGRADE: Supporting parameter sweep applications in workflows. Proc. of the 3rd Workshop on Workflows in Support of Large-Scale Science, Austin (2008)

6. Kertész, A., Kacsuk, P.: GMBS: A New Middleware Service for Making Grids Interoperable. Future Generation Computer Systems 26, 542-553 (2010)

7. Rambadt, M., Weider, P.: UNICORE - Globus: Interoperability of Grid Infrastructures. In: Proceedings of Cray User Group Summit, Manchester (2002)

8. Riedel, M., et al.: Interoperation of World-Wide Production e-Science Infrastructures. Concurrency and Computation: Practice and Experience (2008)

9. Rodero, I., Guim, F., Corbalan, J., Fong, L.L., Liu, Y.G., Sadjadi, S.M.: Looking for an Evolution of Grid Scheduling: Meta-brokering. In: Coregrid Workshop in Grid Middleware 2007, Dresden, Germany (June 2007)

10. Urbah, E., Kacsuk, P., Farkas, Z., Fedak, G., Kecskeméti, G., Lodygensky, O., Marosi, A., Balaton, Z., Caillat, G., Gombás, G., Kornafeld, Á., Kovács, J., He, H., Lovas, R.: EDGeS: Bridging EGEE to BOINC and XtremWeb. Journal of Grid Computing, Special Issue: Grid Interoperability 7(3), 335-354 (2009) 